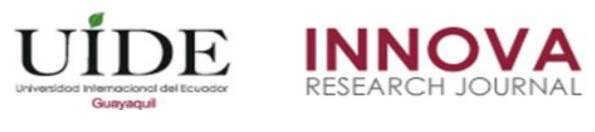

INNOVA Research Journal, ISSN 2477-9024

(Agosto, 2017). Vol. 2, No.8 pp. 86-103

DOI: https://doi.org/10.33890/innova.v2.n8.2017.271

URL: http://revistas.uide.edu.ec/index.php/innova/index

Correo: innova@uide.edu.ec

\title{
El Tapir de montaña (Tapirus pinchaque), como especie bandera en los Andes del sur del Ecuador.
}

\section{The mountain Tapir (Tapirus pinchaque), as a flagship species in the Andes of southern Ecuador.}

\author{
Pedro José Alvarez Loaiza \\ Pablo Antonio Abrigo Cordova \\ Freddy Michael Vite Valverde \\ Daniela Alexandra Trelles Ordóñez \\ Andrea Carolina Espinoza Torres \\ Patricio Yánez M. \\ Universidad Internacional del Ecuador, Ecuador \\ Autor para correspondencia: evalmeida@uce.edu.e,shkireva@unm.edu \\ Fecha de recepción: 23 de Mayo de 2017 - Fecha de aceptación: 10 de Agosto de 2017
}

Resumen: La Carrera de Gestión Turística y Medio Ambiente de la Universidad Internacional del Ecuador Extensión Loja en conjunto con la Fundación de Conservación Jocotoco, desde agosto de 2015, vienen desarrollando actividades de monitoreo y conservación del tapir de montaña (Tapirus pinchaque) en los Andes del sur de Ecuador, específicamente en ambientes de bosque nublado y páramos de la Reserva Biológica Tapichalaca y zonas colindantes. Para ello se propuso la realización del presente proyecto que estableció un sistema de monitoreo de la especie mediante la utilización de cámaras trampa, así como un diagnóstico preliminar y mediante la observación directa de fecas y huellas, con el fin de generar datos poblacionales e imágenes únicas de esta especie en su hábitat natural e identificar los sitios más idóneos para observar estos animales en actividades de ecoturismo; además se creó un cuento didáctico en el que describe los principales aspectos ecológicos del tapir de montaña.

Palabras clave: conservación biológica; especie bandera; tapir de montaña; reserva biológica tapichalaca; parque nacional podocarpus

Abstract: The Tourism and Environmental Management Career of the International University of Ecuador Extension Loja, together with the Jocotoco Conservation Foundation, since August 2015, have been carrying out monitoring and conservation activities of the mountain tapir (Tapirus pinchaque) in the southern Andes Of Ecuador, specifically in environments of cloud forest and páramos of the Biological Reserve Tapichalaca and adjoining zones. For this purpose, the project was proposed to establish a monitoring system of the species through the use of trap cameras, as well as a preliminary diagnosis and through the direct observation of fecas and footprints, in order to generate population data and unique images Of this species in their natural habitat and identify the most suitable sites for observing these animals in ecotourism activities; In addition a didactic tale was created in which it describes the main ecological aspects of the mountain tapir.

Key words: biological conservation; flag species; mountain tapir; tapichalaca biological reserve; podocarpus national park 


\section{Introducción}

Entre ellas, se propuso la realización del presente proyecto con la finalidad de establecer un sistema de monitoreo e investigación del tapir de montaña (Tapirus pinchaque; Figura 1 y 2 ).

La observación de esta especie en su hábitat natural está empezando a ser realizada por empresas de orientación ecoturística como la fundación JOCOTURS.

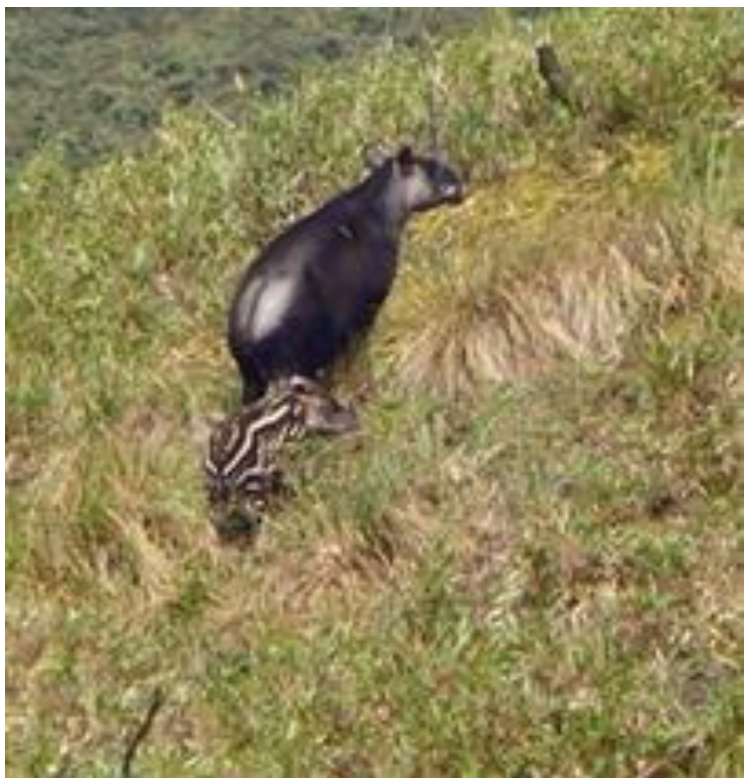

Figura 1. Individuo adulto y cachorro del tapir de montaña (Tapirus pinchaque), septiembre 2016 Fuente: Pedro Álvarez

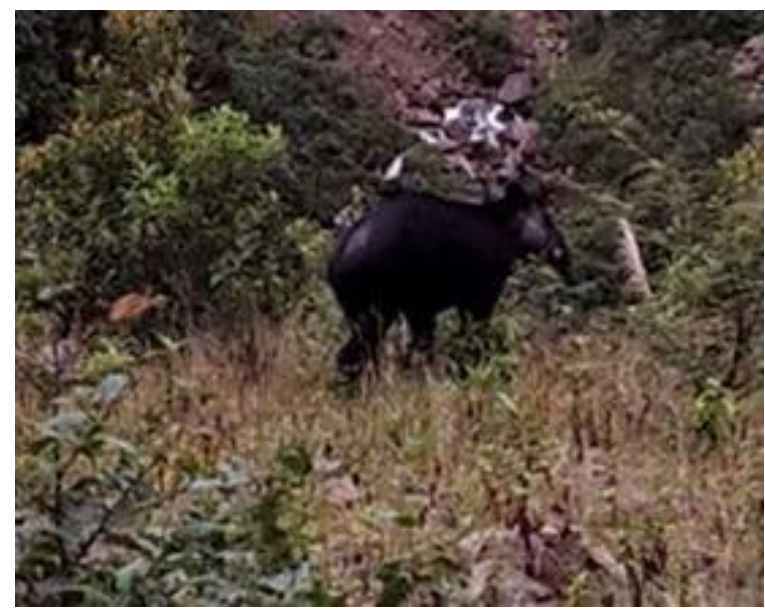

Figura 2. Individuo adulto tapir de montaña (Tapirus pinchaque), acercándose a la quebrada de los muertos "Reserva Tapichalaca" septiembre 2016

Fuente: Pedro Álvarez 


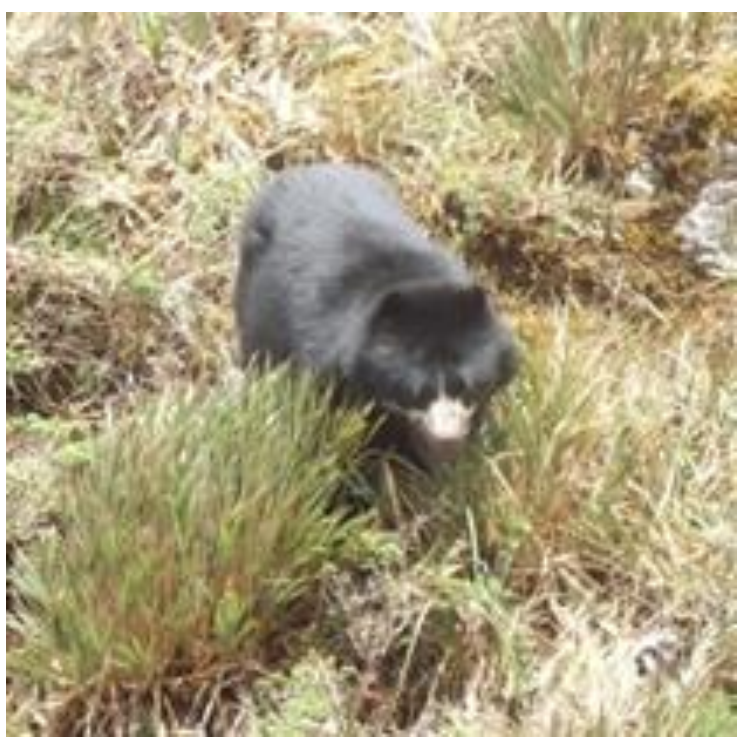

Figura 3. Individuo adulto de oso de anteojos (Tremarctos ornatus), avistado en la zona de paramo, mismo hábitat del tapir de montaña, septiembre 2016 Fuente: Pedro Álvarez

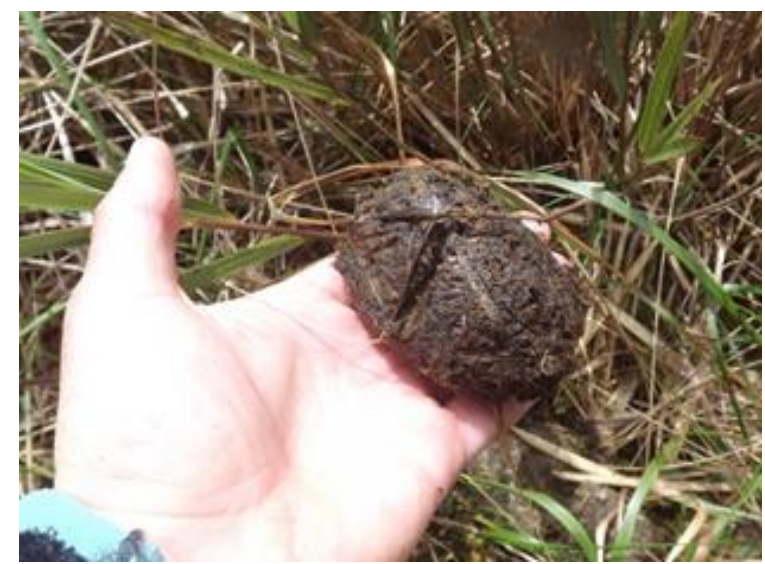

Figura 4. Muestra de feca de (Tapirus pinchaque), avistado en la zona de paramo, mismo hábitat del tapir de montaña, septiembre 2016

Fuente: Pedro Álvarez

Igualmente, al desarrollar el presente proyecto se busca fortalecer actividades lúdicas para efectuar educación ambiental dirigidas a habitantes locales, mediante la elaboración de una cartilla didáctica de la ecología de la especie y complementarlo con la implementación de un sistema de investigación y monitoreo de sus especímenes en sus ambientes naturales de vida, tal como lo recomiendan Castellanos (1994), Brooks \& Eisenberg (1999), Bermudez (2010), Correa \& Torres (2005).

A la vez, se buscó identificar los sitios más idóneos para poder observar ejemplares de esta especie, así como la elaboración de una cartilla o cuento didáctico que describa en términos sencillos los aspectos ecológicos más relevantes de la vida del tapir de montaña.

Las actividades anteriores permitirían delinear propuestas más amplias de conservación de los bosques nublados y páramos del sur del Ecuador, en dónde se tengan reportes de 
avistamientos del tapir de montaña, buscando a la vez un desarrollo sustentable de la actividad ecoturística local, acorde con los objetivos del Plan Nacional del Buen Vivir 2012-2017

(SENPLADES, 2012), los PDOT parroquiales y provinciales; y los Planes de Manejo del Parque Nacional Podocarpus y del Parque Nacional Yacuri

(http://areasprotegidas.ambiente.gob.ec/es/documentos).

Para el desarrollo del presente trabajo investigativo se planteó los siguientes objetivos:

- Establecer un sistema de monitoreo del tapir de montaña (Tapirus pinchaque) en la Reserva Biológica Tapichalaca

- Elaborar una cartilla didáctica sobre la ecología de la especie que permita proponer actividades de conservación.

- Socializar los resultados de la investigación con los actores directos e indirectos.

Las actividades de monitoreo del tapir de la montaña en la reserva Biológica Tapichalaca propondrá actividades de conservación de la especie y fomento del ecoturismo.

Los objetivos e hipótesis de la presente investigación están relacionados directamente por que permitirán utilizar métodos de monitoreo y seguimiento, cuyos resultados al concluir el ensayo establecerán el número de especies detectadas, así como el conocimiento de la especie y su futura conservación.

El problema radica por que no existen mecanismos idóneos de monitoreo, que permitan identificar la abundancia y comportamiento del tapir de la montaña (Tapirus pinchaque), además que sitios son idóneos para desarrollar Ecoturismo en estas áreas protegidas y sus zonas de amortiguamiento. Creando un desarrollo eco turístico sustentable, sobre todo el área de influencia directa de la parroquia Valladolid, basándose en los objetivos el Plan Nacional del Buen Vivir. La noción de elegir esta especie emblemática sostiene que al elevar el perfil de una determinada variedad, es posible obtener más apoyo para la conservación de la biodiversidad en general, en un contexto particular.

\section{Métodos}

\section{Área de estudio}

La presente investigación fue desarrollada en los Andes del sur del Ecuador en ambientes de bosque nublado y páramos de la Reserva Biológica Tapichalaca (Figura 5) ubicados en la Parroquia Valladolid, Cantón Palanda, Provincia Zamora Chinchipe.

El territorio parroquial de Valladolid limita al norte con la parroquia Yangana de la provincia de Loja, en la cordillera de Sabanilla; al sur con la parroquia Palanda hasta la quebrada de Pueblo Viejo, al este con la parroquia El Porvenir del Carmen en el Río Numbala y al oeste con el cantón Espíndola de la provincia de Loja. 


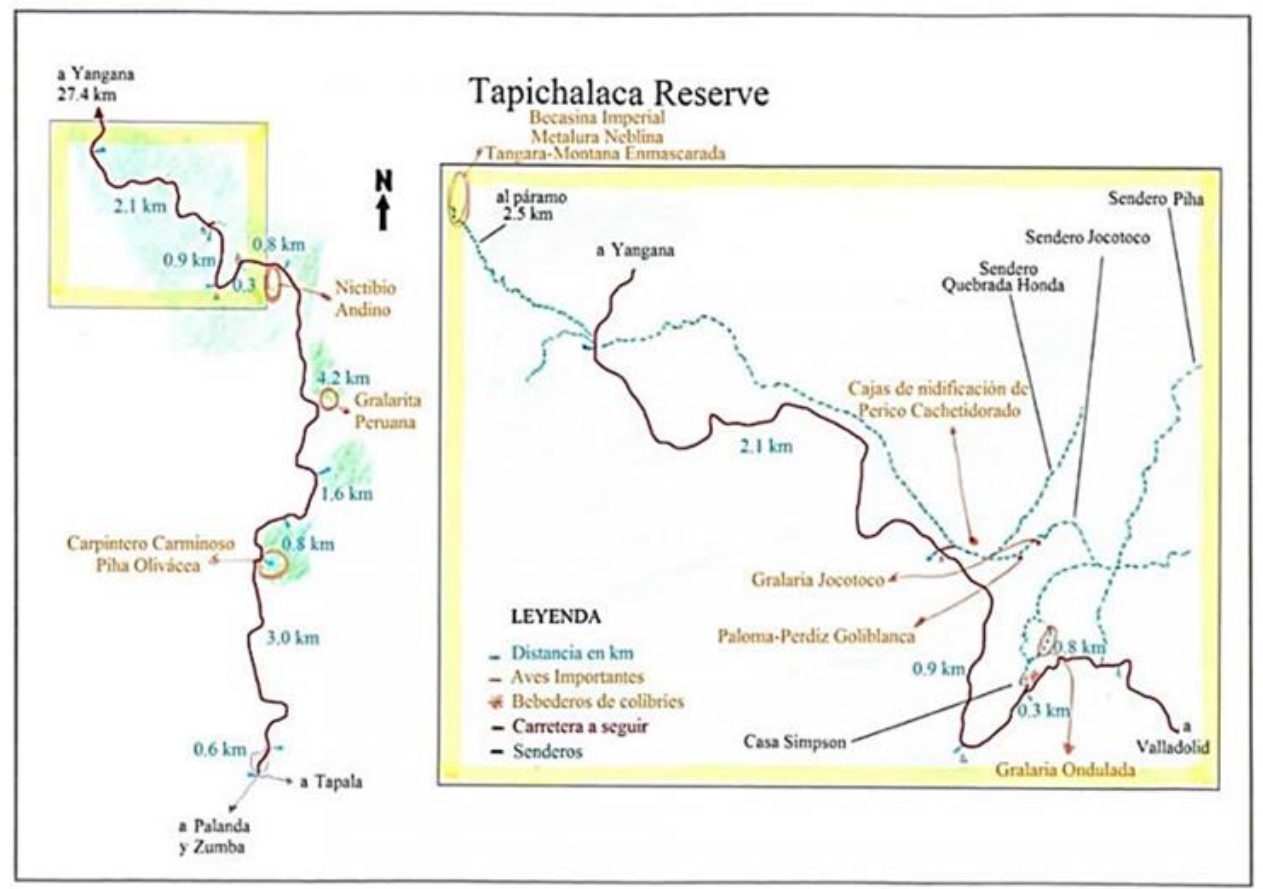

Figura 5. Ubicación del área de estudio (ambientes entre Valladolid y Yangana), enero 2017.

Fuente: Cortesía de la Fundación de Conservación Jocotoco

El área de estudio elegida cuenta con 4 sitios idóneos para desarrollar el ecoturismo, centrado en la observación de mamíferos grandes como son, el oso de anteojos, (Tremarctos ornatus); tapir de montaña (Tapirus pinchaque); lobo o zorro de páramo (Lycalopex culpaeus) y también de aves de páramo o gallinago imperial (Gallinago imperialis); colibrí gigante (Patagona gigas); y otras.

Igualmente, puede constituirse en una zona de observación de cambio de formaciones vegetales desde ambientes de bosque nublado hasta los de páramo (Yánez, 1997) e incluso en una zona de monitoreo de la respuesta de las especies vegetales a eventos de cambio climático (Yánez \& Fariñas, 2000; Yánez \& Picoíta, 2012).

\section{Implementación de las cámaras trampa}

Las cámaras trampa (figura 6) funcionales fueron ubicadas en cuatro zonas diferentes de monitoreo, correspondientes a bosque nublado, páramo y riveras de sistema lacustre paramero. Estos dispositivos nos permitieron registrar información sobre la ecología y abundancia de los tapires, e indirectamente información sobre sitios claves para el desarrollo del ecoturismo para observación de esta especie bandera y de otras típicas de estas zonas andinas. 


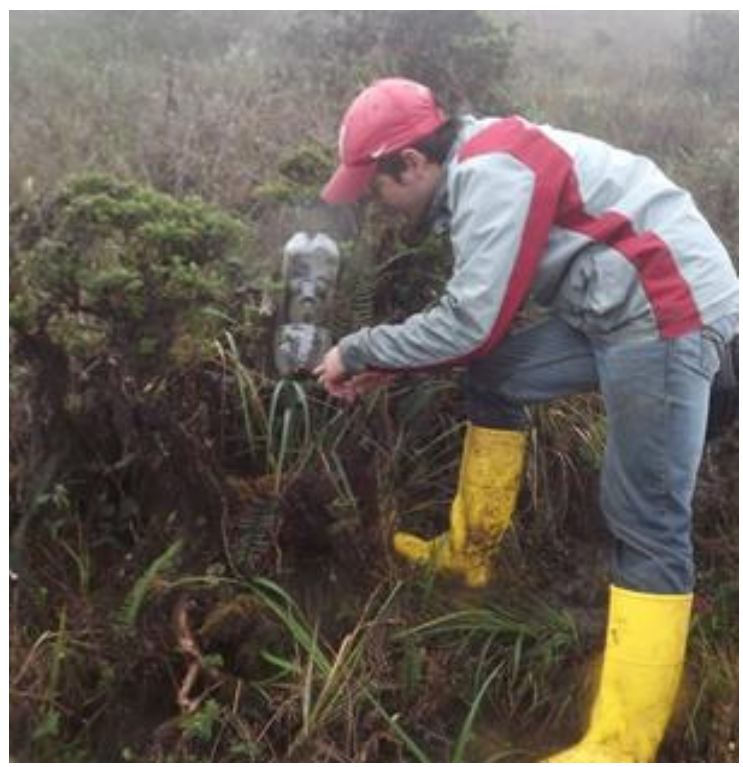

Figura 6. Estudiante de la CGTMA colocando la cámara trampa, bosque nublado II, Reserva Biológica Tapichalaca, mayo 2016.

Fuente: Los autores

\section{Materiales}

\section{Campo:}

- Cámaras trampa (4)

- Memorias card (4)

- Juegos de pilas recargables (4)

- Cámaras digital (1)

- GPS (1)

- Ficha de contenido

- Ficha de registro de atractivos turísticos naturales y culturales

- Fichas de prestadores de servicios

- Fotografías

- Cuestionarios

\section{Oficina:}

- Computadora

- Impresora

Métodos.- Se utilizó la investigación descriptiva, que "buscó especificar las propiedades, las características y los perfiles importantes de personas, grupos, comunidades” (Hernández, 2007).

\section{Técnicas}


Observación Directa: Cercana al sujeto de estudio permitió conocer no sólo el fenómeno sino además el contexto o entorno, logrando que el observador tenga claro el objetivo de la observación, ayudado del registro fotográfico indispensable para el inventario y de notas manuscritas luego de realizado cada periodo de observación.

Grupos focales: Guardaparques de la Reserva Tapichalaca y estudiantes de la carrera de gestión turística y medio ambiente.

Muestreo: Para la investigación de campo se recogió información de fuentes primarias a través de la implementación de planes de muestreos empleando metodologías de cámara trampa distribuidas una o dos cámaras para cada sitio clave y los sitios de muestreo fueron bosque nublado, paramo, y sistema lacustre.

\section{Herramientas}

\section{Diagnósticos}

Las técnicas de investigación documental sirvieron para obtener información de otros autores que han escrito temas relacionados con la investigación. Se revisaron teorías generales y elementos teóricos existentes sobre las variables que se están estudiando. Así mismo, fue necesario analizar la información empírica secundaria o indirecta proveniente de informes de investigación o elaboración de PDOT parroquiales y planes de manejo de áreas protegidas (Parque Nacional Podocarpus, Parque nacional Yacuri, Reserva Biológica Tapichalaca y la reserva parroquial Corazón de la Amazonia) también información de periódicos, revistas científicas especializadas, nos apoyaremos en material producido por el TSG-UICN (grupo de especialistas en tapires; unión mundial para la conservación de la naturaleza): estadísticas generadas, mapas levantados, fotografías, material audio visual y todos aquellos que nos ayuden avanzar con la investigación.

Para el trabajo aplicamos la técnica de fichaje que permitirá extraer y/o sintetizar la información de documentos como libros, revistas, y documentación histórica importante.

El instrumento para el registro la información fue la ficha de citas textuales en donde se anotó en papel la información como una copia textual del tema o contenido consultado.

\section{Resultados y Discusión}

\section{Establecer un sistema de monitoreo del tapir de montaña (Tapirus pinchaque) en la Reserva Biológica Tapichalaca}

Se determinaron, a través del uso de 37 fichas semanales de monitoreo del tapir andino (Tapirus pinchaque) en cuatro sitios idóneos para desarrollar ecoturismo de observación de fauna y flora, centrado en la observación de mamíferos grandes.

Se obtuvo un banco de fotografías de doce meses de investigación y un banco de videos de ocho meses, mismos que a la presente fecha se están procesando. 


\section{Registros de abundancia del tapir de montaña}

En la Tabla 1 se puede observar el número de registros de presencia de tapir de montaña en los 4 sitios de monitoreo.

Tabla No. 1. Registros de presencia de tapir de montaña (por observación directa de ejemplares, huellas, rastros) en cuatro sitios de observación efectuados en el presente estudio

\begin{tabular}{|c|c|c|c|c|}
\hline \multirow[b]{2}{*}{ Mes de registro } & \multicolumn{2}{|c|}{ Código del sitio } & \multirow[b]{2}{*}{$\begin{array}{l}\text { Páramo } \\
\text { P1 }\end{array}$} & \multirow[b]{2}{*}{$\begin{array}{l}\text { Laguna } \\
\text { de Páram } \\
\text { LP1 }\end{array}$} \\
\hline & $\begin{array}{l}\text { Bosque } \\
\text { Nublado } \\
\text { BN1 }\end{array}$ & $\begin{array}{l}\text { Bosque } \\
\text { Nublado } \\
\text { BN2 }\end{array}$ & & \\
\hline Septiembre 2015 & 0 & 0 & 2 & 5 \\
\hline Octubre 2015 & 0 & 0 & 0 & 6 \\
\hline Noviembre 2015 & 0 & 0 & 0 & 4 \\
\hline Diciembre 2015 & 2 & 4 & 0 & 6 \\
\hline Enero 2016 & 8 & 10 & 2 & 4 \\
\hline Febrero 2016 & 10 & 4 & 1 & 0 \\
\hline Marzo 2016 & 12 & 8 & 0 & 2 \\
\hline Abril 2016 & 8 & 12 & 1 & 0 \\
\hline Mayo 2016 & 4 & 6 & 0 & 1 \\
\hline Junio 2016 & 2 & 2 & 0 & 2 \\
\hline Julio 2016 & 2 & 4 & 2 & 4 \\
\hline Agosto 2016 & 2 & 2 & 2 & 2 \\
\hline Total de registros de presencia del tapir de montaña & 50 & 52 & 10 & 36 \\
\hline Promedio de registros por mes & 4,17 & 4,33 & 0,83 & 3,00 \\
\hline Mediana de registros por mes & 2,00 & 4,00 & 0,50 & 3,00 \\
\hline
\end{tabular}

Elaboración: Pedro Álvarez

Fuente: Pedro Álvarez

Con los registros de observación de los cuatro sitios se utilizó el Análisis de Varianza no Paramétrico (Kruskal-Wallis), de acuerdo a lo recomendado por Yánez (2005) cuando no se detecta distribución normal en los juegos de datos a ser comparados. El estadístico $\mathrm{H}$ fue de 8,$87 ; \mathrm{p}=0,03$. Esto permitió distinguir que los sitios BN1, BN2 y LP1 son significativamente diferentes a $\mathrm{P} 1$.

BN1, BN2 y LP1 son sitios en los que la probabilidad de observar tapires de montaña es mayor a lo largo del año, y constituirían, al menos de manera preliminar, sitios en los cuales se podría realizar la observación de este tipo de fauna en la zona de estudio a través de actividades de ecoturismo planificadas (sensu Conservation Tourism, 2010), considerando el avistamiento a distancias razonables y con el uso de largavistas para no causar ningún tipo de stress en estos animales, tal como lo recomiendan algunos expertos en la conservación del tapir de montaña (Downer, 1996; Downer, 2001; Downer \& Castellanos, 2002). 
A continuación se presenta el grafico 1, donde se observa el movimiento del tapir desde el mes de septiembre 2015 hasta julio 2016.

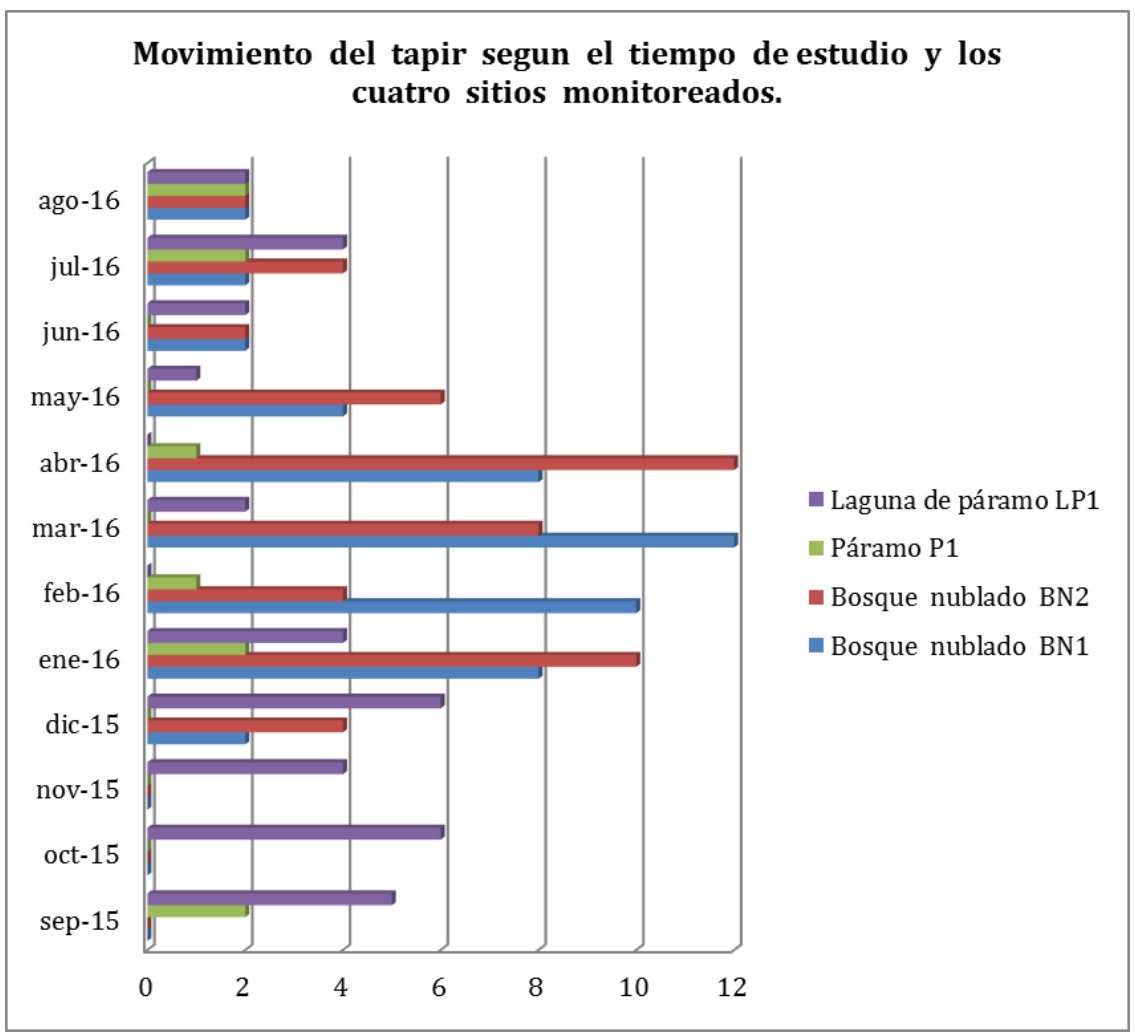

Grafico No. 1. Registros de presencia de tapir de montaña (por observación directa de ejemplares, fecas y huellas o rastros) en los ecosistemas paramo, laguna o lacustre y bosque nublado.

Fuente: Autores

Elaboración: Autores

Se evidencia que el sitio donde más registros se obtuvo del tapir de montaña, es bosque nublado con individuos observados directamente, tanto BN1 con 50 y BN2 con 52 individuos, de acuerdo al gráfico, evidenciando mayor flujo en estos sitios y los meses donde más movimientos hay son desde marzo a mayo y desde octubre a diciembre mucha incidencia en el páramo.

\section{Elaborar una cartilla didáctica sobre la ecología de la especie que permita proponer actividades de conservación.}

Para diseñar el cuento didáctico se procedió a compilar información como parte de un componente para efectuar programas de educación ambiental a futuro en zonas de amortiguamiento de los andes del sur. Con este resultado tomamos en cuenta la ecología y comportamiento del tapir, así como también todas las experiencias adquiridas en el campo que fueron inspiración para crear el cuento, los créditos de las ilustraciones, texto y elaboración de la cartilla que se detalla a continuación. 


Soy el mamifero más grande del Eauador, me enaventro criticamente (CR) amenazado de oxtinción, mi cosa es la coordillera de los Andes.

Especialmente la vertionte horionaal.

Aos en el sur vivo en la resenva de biosfera Pocbcorpus El conclor

Say uno de los mamiferos más grandes criticamente amenazados on al mundo, me enachtro dentro de areos protegidas oen lugares de díficil acoess, grandes extenciones de mi hóbitatớriginal han sido reemplazados por la daricalltura, ganaderia, areas urboras y bosques intruducídos.

comporto mi hóbitat con el tugan, el 050 de antegíos, el lobo de poramo el colibri y otros amigos más.
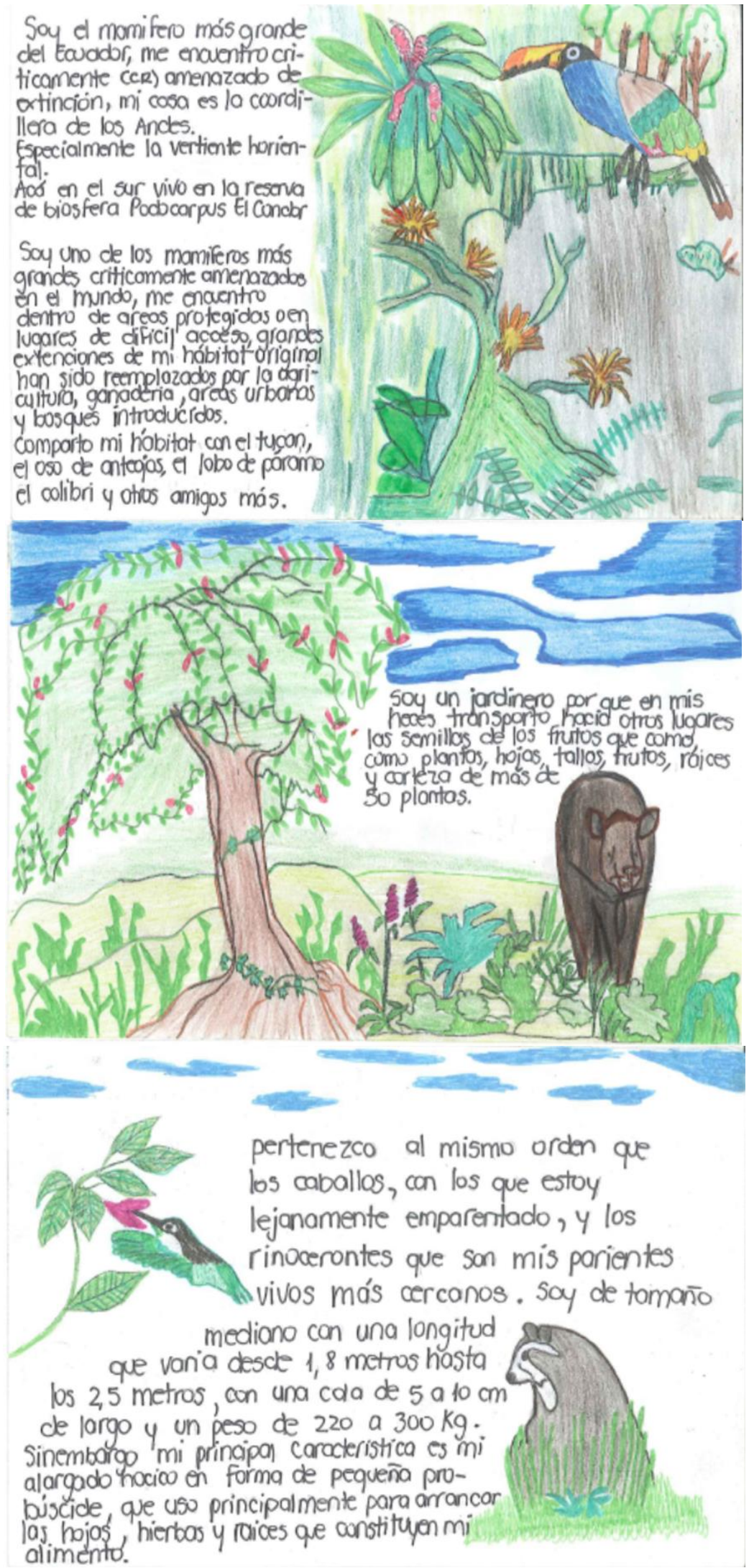


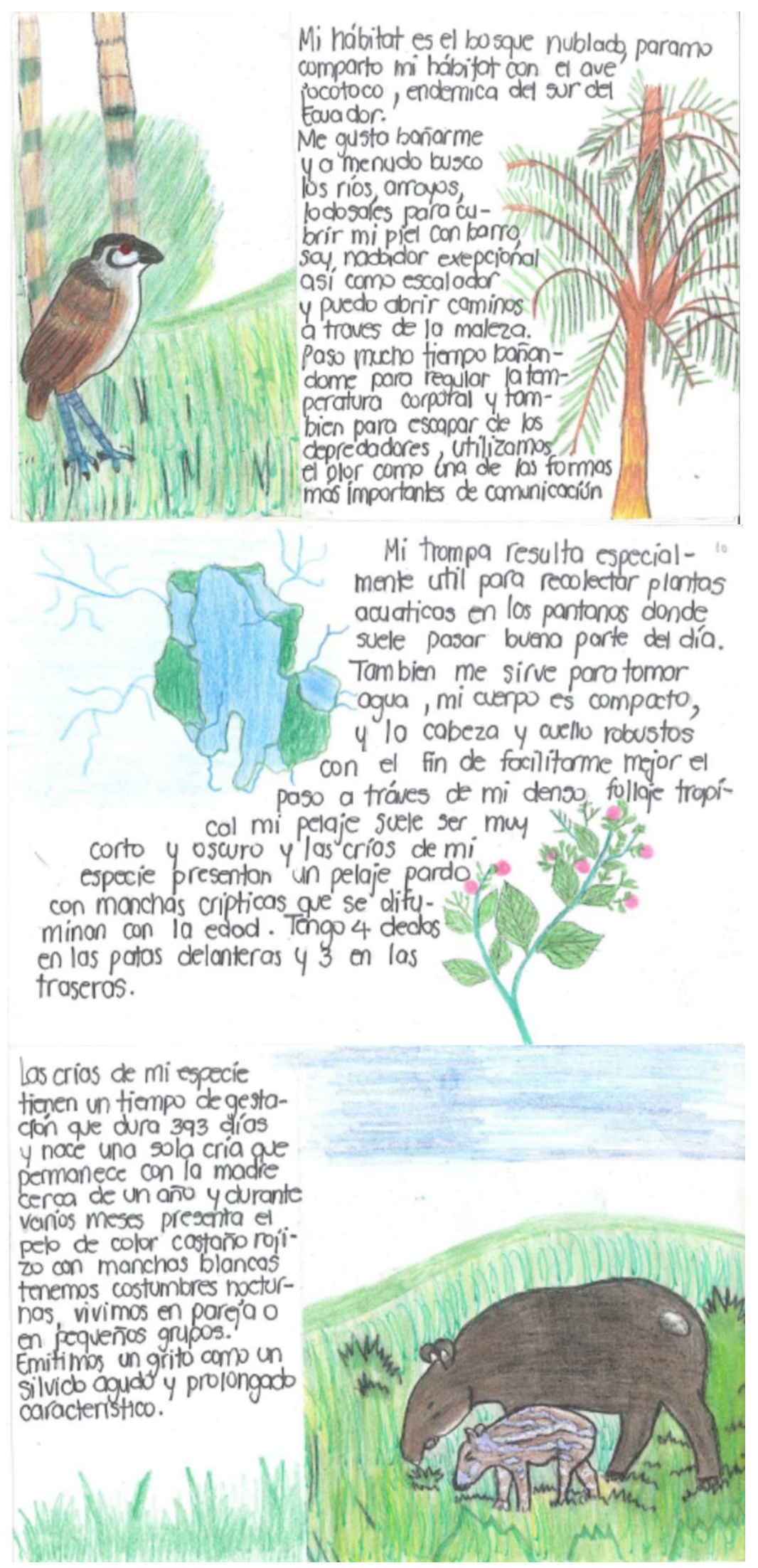




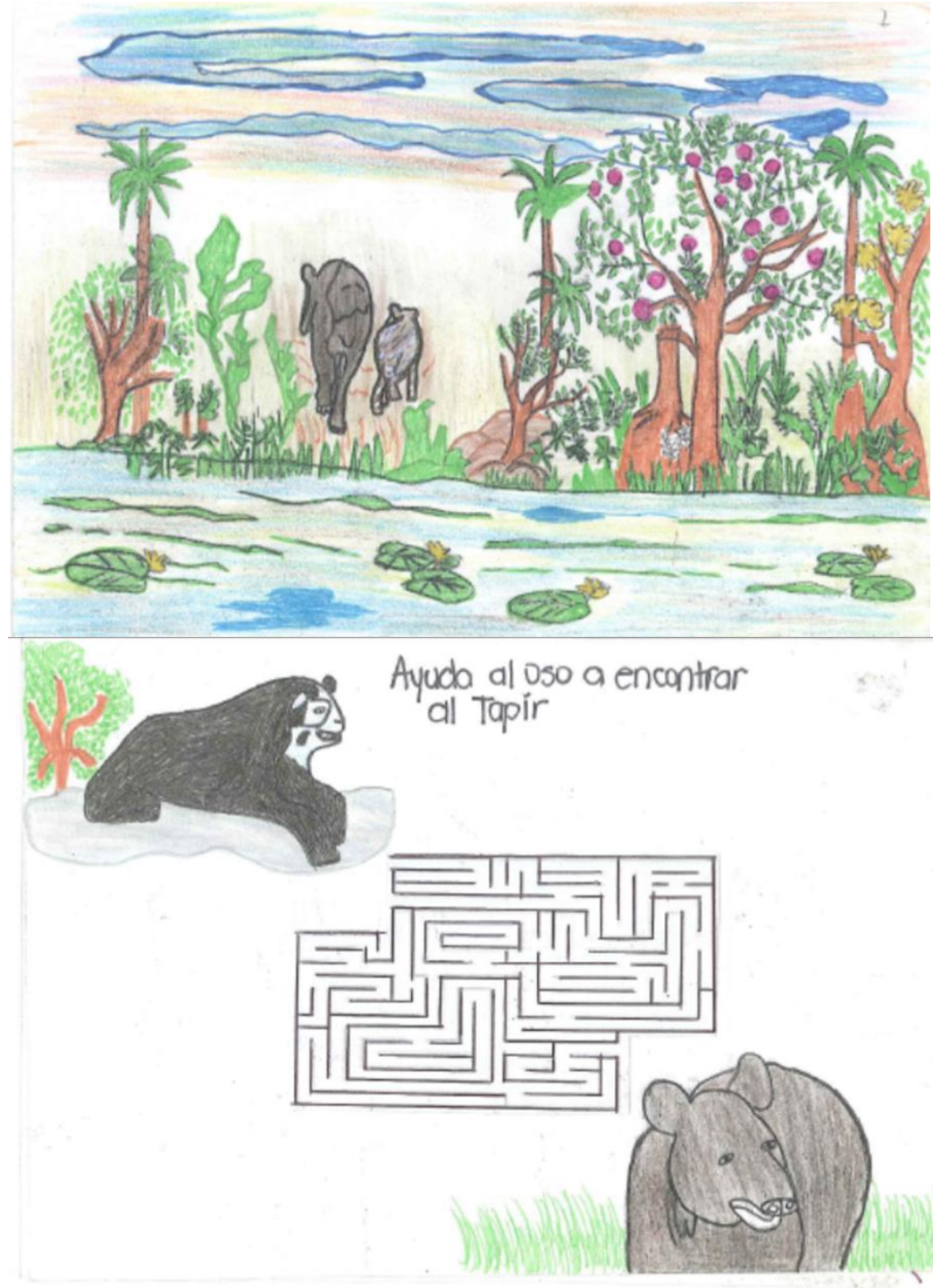

Sigue el camino del bosque nublado

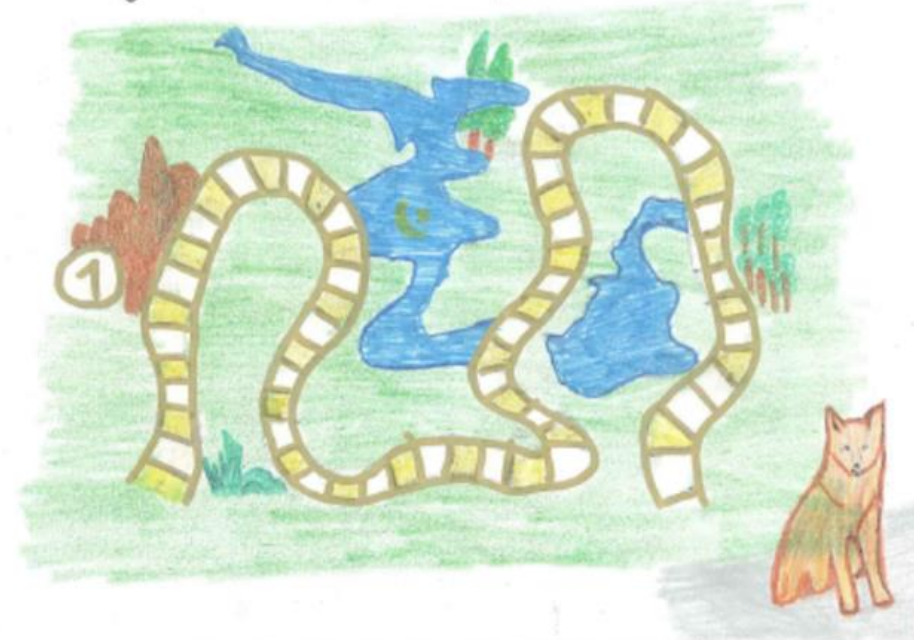




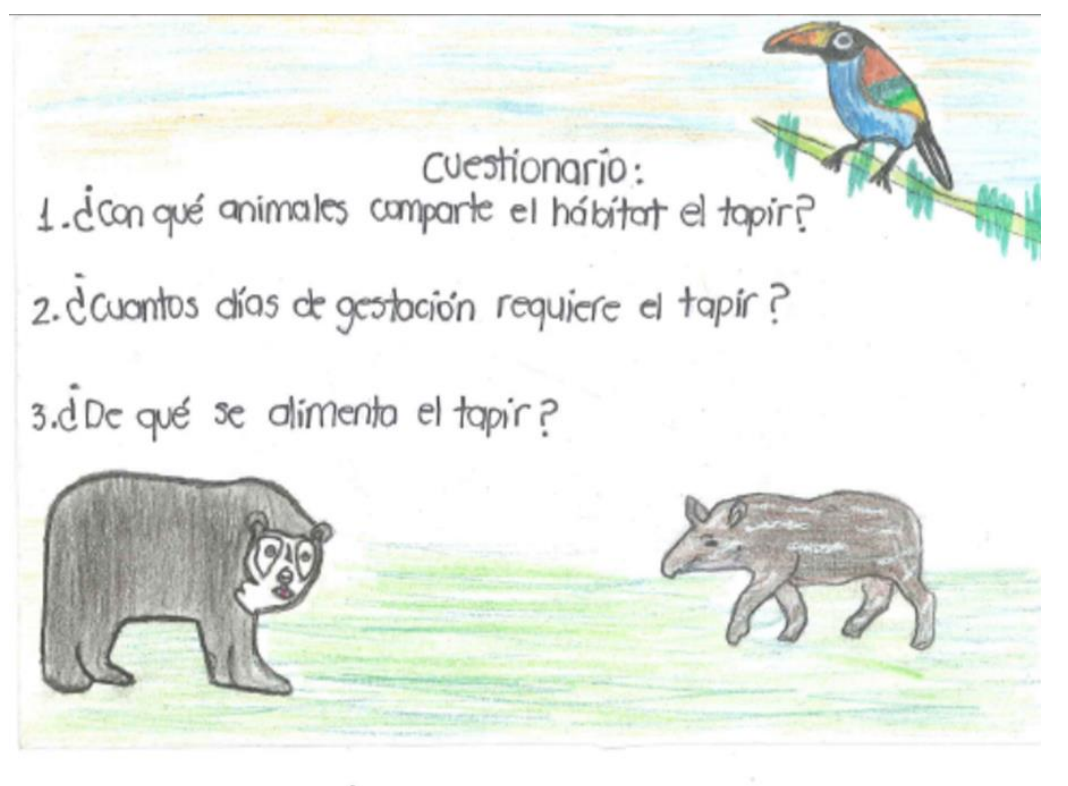

Fuente: Ing. Andrea Carolina Espinoza Torres

Elaboración: Ing. Andrea Carolina Espinoza Torres

\section{Socializar los resultados de la investigación con los actores directos e indirectos}

La socialización de los resultados del proyecto se realizó en dos sitios: El primero en las instalaciones de la Universidad Internacional del Ecuador extensión Loja, donde asistieron 33 estudiantes. El segundo evento se realizó en el colegio Ciudad de Loyola perteneciente a la parroquia Valladolid, donde asistieron 60 personas entre docentes y estudiantes.

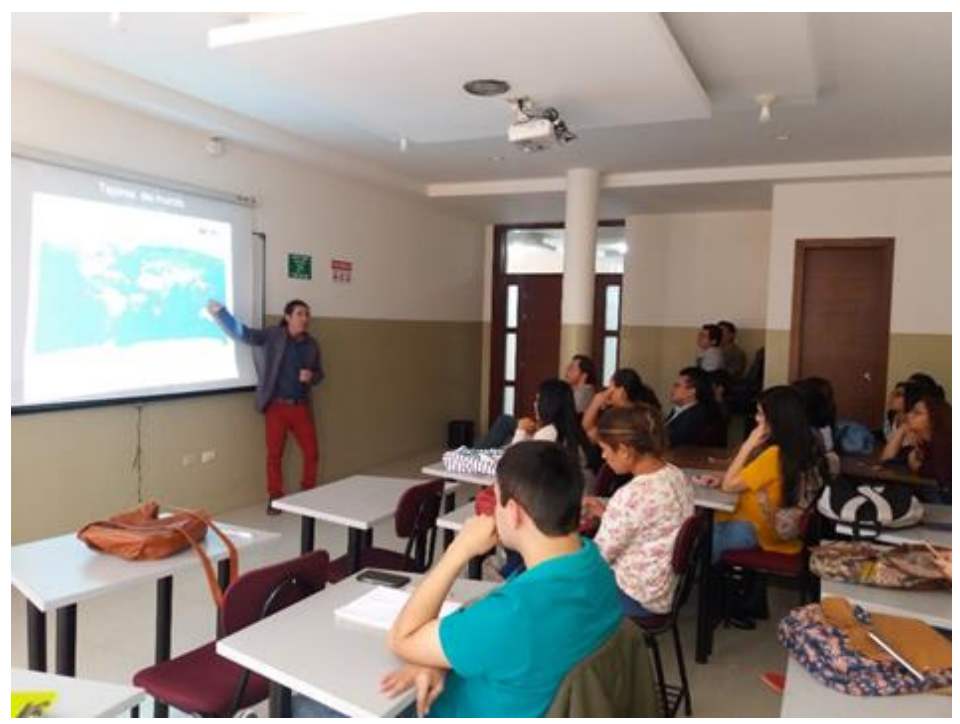

Figura 7, Socialización de los resultados de la investigación por intermedio del Mgs. Pedro Álvarez, 20 de octubre de 2016.

Fuente: Autores 


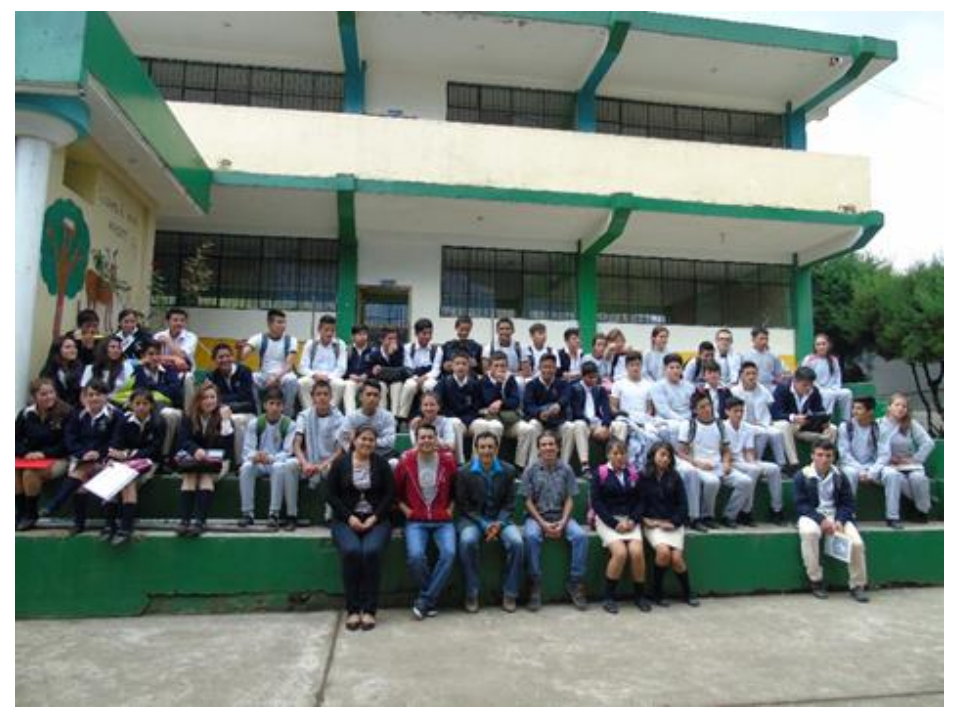

Figura 8, Socialización de los resultados de la investigación por intermedio de los Mgs. Pedro Alvarez, Pablo Abrigo, Michael Vite, Daniela Trelles, 20 de octubre de 2016

Fuente: Autores

\section{Recomendaciones}

Se recomienda que se continúe recabando información de aspectos ecológicos poblacionales y eco sistémicos de Tapirus pinchaque en la zona de trabajo y áreas contiguas, para avanzar con la iniciativa de desarrollar actividades de conservación de la especie y desarrollo ecoturístico en la zona.

Actividades de ecoturismo, turismo comunitario y de educación ambiental deben estar articuladas en un plan a mediano o largo plazo, siempre aplicando el uso cuidadoso del recurso paisajístico andino local, y la consideración de la especie bandera Tapirus pinchaque, en torno a la cual giraría potencialmente la planificación del desarrollo de la actividad turística-educativa.

\section{Conclusiones}

En general, podemos mencionar que el tapir de montaña es una especie relativamente difícil de observar de manera directa, pero es un importante indicador de buena conservación de un hábitat andino, y a la vez puede atraer viajeros y turistas de naturaleza de diferentes partes del mundo.

En el presente estudio se han determinado cuatro zonas de avistamiento de tapires en la Reserva Tapichalaca, entre de las cuales (dos de bosque nublado y una laguna paramera) se tiene una alta probabilidad de observación de estos animales a lo largo del año.

El avistamiento de estos animales amerita cierta preparación física en los visitantes, y una capacidad media para efectuar trekking en ambientes montañosos. Por lo que se puede concluir que en los senderos locales se puede desarrollar ecoturismo y también un turismo alternativo o de aventura. 
Se tienen bien registrados los datos de los sitios de avistamiento, esto permitirá sugerir al personal de la Reserva Tapichalaca que se los cuide y monitoree de manera planificada.

La idea de desarrollar una cartilla y/o cuento didáctico para la zona, es que se efectué como un componente de educación ambiental en las zonas de amortiguamiento del estudio, es decir, en las parroquias Valladolid y Yangana respectivamente. 


\section{Bibliografía}

\section{Fuentes impresas:}

Bermúdez, D. (2010). Dieta y hábitos alimenticios del Tapir Andino (T. pinchaque) entre localidades del Corredor Ecológico Llanganates - Sangay. Quito: Universidad Central del Ecuador, Tesis de Licenciatura.

Brooks, D., \& Eisenberg, J. (1999). Estado y biología de los tapires neotropicales: perspectiva general. Manejo y conservación de fauna silvestre en América Latina, 409-414.

Castellanos, A. (1994). El tapir andino (Tapirus pinchaque Roulin): crianza de un ejemplar en el Bosque Protector Pasochoa y notas ecológicas en el Parque Nacional Sangay, Ecuador. Quito: Universidad Central del Ecuador, Tesis de Licenciatura.

Correa, A., \& Torres, N. (2005). Estrategia nacional preliminar para la conservación de los tapires (género Tapirus) en el Ecuador. Loja: Universidad Técnica Particular de Loja, Tesis de Licenciatura.

Downer, C. (1996). The Mountain Tapir, endagered "flagship" species of the high Andes. Oryx, 30, 45-58.

Downer, C. (2001). Observations on the diet and habitat of the mountain tapir (Tapirus pinchaque). J. Zool. Lond., 254, 279-291.

Downer, C., \& Castellanos, A. (2002). Tapirus pinchaque. En: IUCN Red List of Threatened Species.

Nasimba, C., \& Cejas, M. (2015). Diseño de productos turísticos y sus facilidades. Qualitas, 10, 22-39.

Ridgely R \& Greenfield P. (2006). Aves del Ecuador. Quito Ecuador.

SENPLADES. (2012). Plan Nacional del Buen Vivir. Quito.

Tirira, D. G. ed. (2011). Libro rojo de los mamíferos del Ecuador . 2a. ed. Fundacion mamiferos y conservacion

Yánez, A. P. (1997a). Seminario Bibliográfico “Distribución de especies vegetales en ecosistemas naturales desde la óptica del Continuum: Evolución de la Idea y algunos Métodos para su Caracterización”. CIELAT, Universidad de Los Andes, Mérida, Venezuela, 44pp.

Yánez, A. P. (1997b). Análisis de la distribución de especies vegetales a lo largo de un gradiente altitudinal Páramo-Selva Nublada del Parque Nacional Sierra Nevada, Venezuela. Tesis de Maestría en Ecología Tropical. Universidad de Los Andes. Mérida, Venezuela. 
Yánez, P., \& Fariñas, M. (2000). Estimación de óptimos de distribución de especies vegetales en un gradiente altitudinal andino y su relación con el monitoreo futuro de estos ambientes. Memorias del IV Congreso Latinoamericano de Ecología. Arequipa: UNSA.

Yánez, P. (2005). Biometría y Bioestadística fundamentales. Analizando la estructura numérica de la información en proyectos ecológicos. Quito.

Yánez, P., \& Picoíta, F. (2012). Posibles efectos del cambio climático global en ambientes andinos de bosque montano y páramo de la sierra centro de Ecuador. Quito: UNIBE.

Yánez, P. (2014a). Ecología y biodiversidad: un enfoque desde el neotrópico. Quito: UNIBE/UIDE. 172pp.

Yánez, P. (2014b). Un vistazo a la conservación Biológica del Ecuador. Qualitas, 7, 80-84.

Ridgely R \& Greenfield P. 2006. Aves del Ecuador. Quyito Ecuador.

\section{Fuentes virtuales:}

Abrigo, P. A. A. (2016). Identificación de Necesidades para Proponer el Desarrollo Turístico en la Comunidad de Tuncarta, del Cantón Saraguro de la Provincia de Loja. INNOVA Research Journal, 1(6).

Conservation Tourism. (2010). Wallingford, GB: CAB International. Retrieved from http://www.ebrary.com

http://areasprotegidas.ambiente.gob.ec/es/documentos Fecha de consulta: 20 de diciembre 2016.

https://upload.wikimedia.org/wikipedia/commons/2/21/Tapirus_pinchaque_portrait.jpg Fecha de consulta: 15 de diciembre 2016.

IUCN. 2008. 2008 IUCN Red list of Theatered Species. www.Iucnredlist.org 